\title{
Simulation of Route Optimization in Mobile IP
}

\author{
Hao Chen and Ljiljana Trajković \\ Simon Fraser University \\ Burnaby, BC, Canada V5A 1 S6 \\ $\{$ lcheu,ljilja\}@cs.sfu.ca
}

\begin{abstract}
Mobile Internet Protocol has been proposed by IETF to support portable IP addresses for mobile devices that often change their network access points to the Internet. In the basic mobile IP protocol, datagrams sent from wired or wireless hosts and destined for the mobile host that is away from home, have to be routed through the home agent. Nevertheless, datagrams sent from mobile hosts to wired hosts can be routed directly. This asymmetric routing, called "triangle routing," is often far from optimal and "route optimization" has been proposed to address this problem. In this paper, we present the implementation of "route optimization" extension to mobile IP in the ns-2 simulator. We illustrate simulations of the mobile IP with route optimization with simulation scenarios, parameters, and simulations results.
\end{abstract}

\section{Mobile IP}

We first provide an overview of the mobile Internet Protocol (MIP) [1], including the "triangle routing" problem and the route optimization [2] in mobile IP.

Mobile IP, the mobility support for IP, enables a mobile host $(\mathrm{MH})$ to send datagrams to the correspondent host $(\mathrm{CH})$ directly, routed by its home agent $(\mathrm{HA})$ and foreign agent (FA) (Figure 1). However, packets from $\mathrm{CH}$ to $\mathrm{MH}$ have to be routed through three different (sub)networks: the CH's subnet, the HA's subnet, and the FA's subnet where the MH is currently located. Therefore, packets destined to the $\mathrm{MH}$ are often routed along paths that are significantly longer than optimal. This redundant routing in mobile IP is known as "triangle routing." Route optimization addresses this problem by requiring all hosts to maintain a binding cache containing the care-of address of MHs. The binding cache is a cache of mobility bindings of mobile nodes, maintained by a node to be used in tunneling datagrams to mobile nodes. Route optimization extension to mobile IP includes four messages: binding update, binding warning, binding request, and binding acknowledgment. A binding update message is used to inform the $\mathrm{CH}$ of the MH's current mobility binding. The binding warning message is used to transmit warnings that a binding update message is needed by one or more correspondent hosts.

We employed $n s-2$ network simulator $[3,4]$ to implement the route optimization extension in mobile IP. Two (out of four) route optimization messages have been implemented: binding update and binding warning. We have compared end-to-end packet delays in the basic routing scheme in mobile IP with and without optimization. We show that mobile IP with the route optimization has smaller end-to-end packet delay than the basic mobile IP.

\section{Simulation scenarios and results}

The simulation scenario is shown in Figure 1. It consists of one mobile host, one home agent, two foreign agents, one correspondent host, and one wired node representing a public network (PN).

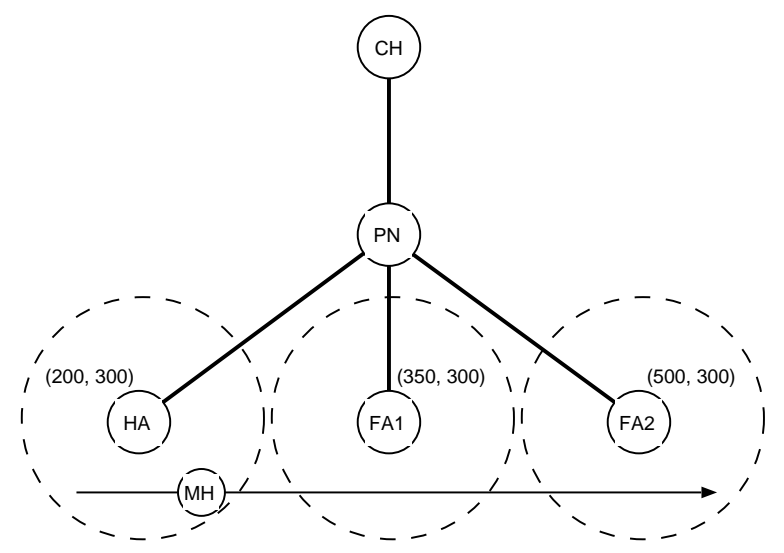

Figure 1. Simulation scenario with two foreign agents.

We use simulation results to verify the effectiveness of our implementation. End-to-end packet delay and average 
end-to-end packet delay with (solid) and without (dashed) route optimization in mobile IP are shown in Figures 2 and 3 , respectively. We can observe that packets loss begins at approximately $35 \mathrm{sec}$, when MH moves out of the wireless home network. Packet flow resumes when the MH successfully registers in the foreign network FA1. Packet loss occurs again at approximately $65 \mathrm{sec}$ when the MH leaves foreign network FA1 and enters foreign network FA2. Packet flow resumes again at approximately $70 \mathrm{sec}$.

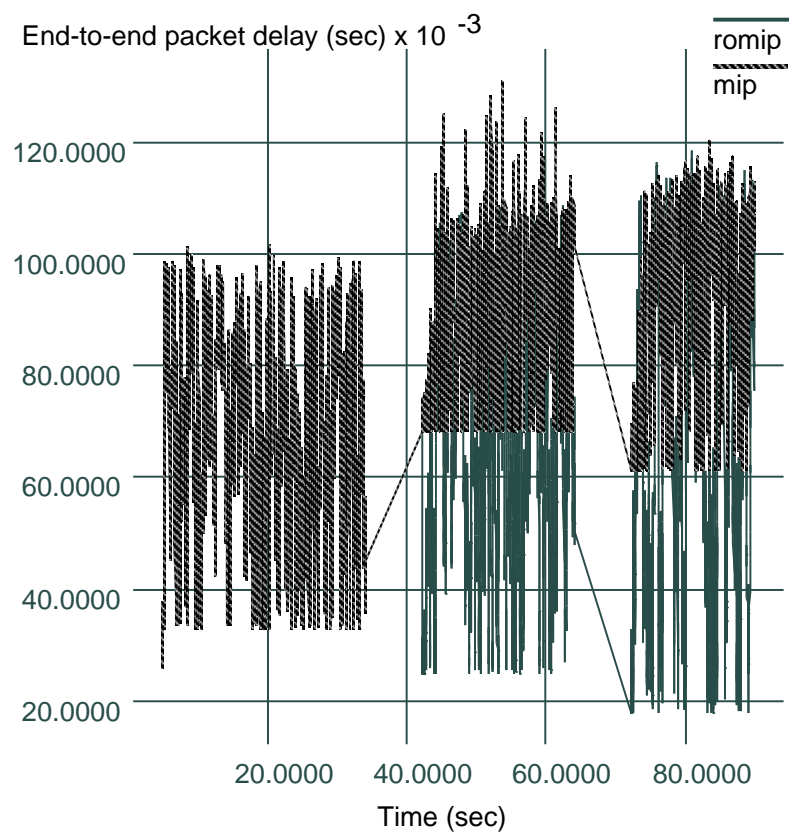

Figure 2. End-to-end packet delay with (solid) and without (dashed) route optimization in mobile IP.

With the route changes, the end-to-end packet delays change accordingly. In the case of mobile IP without route optimization, we notice that when the $\mathrm{MH}$ is in the foreign network, the end-to-end packet delay is always larger than the delay when $\mathrm{MH}$ is in the home network, no matter what the link delay is from the $\mathrm{CH}$ to the $\mathrm{MH}$. When the $\mathrm{MH}$ moves to the foreign network, the minimum end-toend packet delay with route optimization (shown in Figure 2 ) is much smaller than the end-to-end packet delay without route optimization. When the MH moves to the foreign network, the average end-to-end packet delay, shown in Figure 3 , retains the same level as the average delay when the $\mathrm{MH}$ remains in the home network.

These simulation results illustrate the effectiveness of route optimization in the mobile IP protocol. The network efficiency and utilization have been improved by eliminating the "triangle routing."

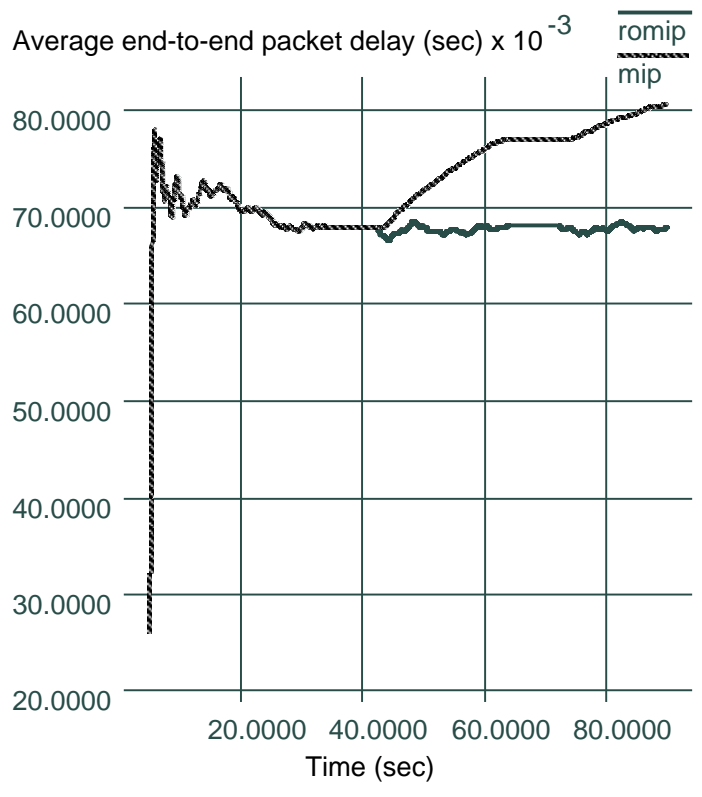

\section{Figure 3. Average end-to-end packet delay with (solid) and without (dashed) route op- timization in mobile IP.}

\section{Summary}

In this paper, we considered route optimization in mobile IP. We modified $n s-2$ and extended the mobile IP packets to enable the route optimization. Simulation results verified the effectiveness and efficiency of route optimization in mobile IP. Although we implemented only two route optimization messages, our implementation proved effective and sufficient to demonstrate the significance of route optimization in mobile IP. IPv6, the next generation IP, has already integrated "route optimization" as a fundamental part of the mobility support [5].

\section{References}

[1] C. Perkins, IP mobility support for IPv4: http://www.ietf.org/rfc/rfc3220.txt (January 2002).

[2] P. Zhou, and W. Yang, "Reverse routing: an alternative to MIP and ROMIP protocols," in Proc. Canadian Conference on Electrical and Computer Engineering, Edmonton, AB, Canada, May 1999, vol. 1, pp. 150-155.

[3] The network simulator - ns-2: http://www.isi.edu/nsnam/ns (August 2002).

[4] K. Fall and K. Varadhan (Eds.), The ns Manual: http://www.isi.edu/nsnam/ns/doc/index.html (August 2002).

[5] C. Perkins, Mobile IP: Design Principles and Practices. Reading, MA: Addison-Wesley, 1998. 